KOBE-TH-03-04

\title{
Escape from washing out of baryon number in a two-zero-texture general Zee model compatible with the large mixing angle MSW solution
}

\author{
K. Hasegawa:* C. S. Lim $\#$ and K. Ogur $\sharp$ \\ Department of Physics, Kobe University, \\ Rokkodaicho 1-1, Nada ward, Kobe 657-8501, Japan
}

(Dated: May 26, 2022)

\begin{abstract}
We propose a two-zero-texture general Zee model, compatible with the large mixing angle Mikheyev-Smirnov-Wolfenstein solution. The washing out of the baryon number does not occur in this model for an adequate parameter range. We check the consistency of a model with the constraints coming from flavor changing neutral current processes, the recent cosmic microwave background observation, and the Z-burst scenario.

PACS numbers: 14.60.Pq,13.60.Rj,26.65.+t
\end{abstract}

Keywords: Zee model, LMA-MSW, Leptogenesis

*kouhei@phys.sci.kobe-u.ac.jp

llim@phys.sci.kobe-u.ac.jp

ogure@phys.sci.kobe-u.ac.jp 


\section{INTRODUCTION}

Recent neutrino oscillation experiments reveal that neutrinos have small but finite mass differences 1, 2, 3]. The precise observation of the cosmic microwave background (CMB) shows that not only the mass differences, but also the masses themselves, are small 4, 5, 6, 7, 8, 9]. The smallness of the neutrino mass is naturally explained in the seesaw mechanism [10, 11, 12] with right-handed neutrinos and in the Zee model with radiative mass generation [13]. In the present paper, we focus on the Zee model, which has many predictions for low energy physics. This model has two Higgs doublets and a singlet Higgs scalar field (the Zee singlet), in addition to the fields in the standard model. In the original Zee model, both of the two Higgs doublets couple to the ordinary matter fields. Wolfenstein, however, imposed a discrete symmetry on the Zee model so that only one of the two Higgs doublets couples to the ordinary matter fields. As a result, dangerous processes through the flavor changing neutral current (FCNCs ) are easily avoided in this model, and this simplified model has been mainly considered so far [14]. We call this model the restricted Zee model (RZM) [15]. One of the attractive points of the Zee model in this version is that the bilarge mixing angle pattern, with respect to the solar and atmospheric neutrino oscillations, is naturally explained. This attractive feature, ironically, has forced the Zee model into a difficult position very recently. The predicted large angles must be just around $\pi / 4$ [16, 17, 18] and are incompatible with the large mixing angle (LMA) Mikheyev-SmirnovWolfenstein (MSW) solution with $\theta_{\odot} \sim \pi / 5.6$, while the KamLAND experiment reports that only the LMA MSW solution is allowed [3]. Several ideas have been proposed to escape from this difficulty: introducing a fourth neutrino [19, 20], introducing a doubly charged Higgs scalar field [21], introducing triplet Higgs scalars [22, 23, 24], and allowing both of the two Higgs doublets to couple to the ordinary matter fields.

Aside from the difficulty concerning the solar mixing angle, we have another issue with this model in cosmology. Since the lepton number is violated in this model, the primordial baryon number is washed out through the sphaleron process at quite a low temperature [25, 26]. This model therefore seems to be unable to exist together with baryogenesis scenarios in which the baryon number is produced at a high temperature. However, it has been pointed out that there exists an approximately conserved number $L^{\prime} \equiv L_{e}-L_{\mu}-L_{\tau}$ for the low mass, low probability (LOW) and vacuum oscillation (VO) solutions of the solar neutrino 
problem in the RZM, and complete baryon number washing out does not occur [27].

In the present paper, we focus on the original Zee model in which both of the two Higgs doublets couple to the ordinary matter fields. We call this model the general Zee model (GZM) in order to clearly distinguish it from the RZM [15]. Although this model was proposed earlier, it has not been extensively discussed, as we mentioned above. We shed light on the GZM once again and find not only that the two phenomenological problems above can be avoided [58] but also that the GZM can be consistent with experiments involving FCNCs. This model is attractive in the sense that the extension from the RZM, of which properties have been extensively discussed, is minimal. Although the extension is minimal, many undetermined parameters arise and the investigation in the full parameter region is very difficult. We then constrain the structure of the neutrino mass matrix obtained from the Zee model. Two constraints for the neutrino mass matrix have been proposed: imposing a texture to have two independent zeros in the base, where the masses of the charged leptons are diagonalized [31], and requiring the mass matrix to have a vanishing determinant 32]. While the vanishing determinant constraint is good in that the condition does not depend on the weak base we take, and it is suitable for an investigation into the Zee model with a doubly charged Higgs singlet [33, 34], it does not fit the structure of the mass matrix obtained from the Zee model considered here. We therefore employ the two-independent-zero-texture constraint, of which phenomenological aspects were investigated in Refs. [35, 36, 37]. The suitable two-zero-texture mass matrix for the Zee model considered here is the case C defined in Ref. [31],

$$
\mathcal{M}_{\nu}=\left(\begin{array}{ccc}
\times & \times & \times \\
\times & 0 & \times \\
\times & \times & 0
\end{array}\right) .
$$

The main aim of the present paper is to search for the simplest GZM with this structure of the mass matrix, which is compatible with the LMA MSW solution of the solar neutrino problem and free from the baryon number washing out [59]. It turns out that such a GZM can be constructed by minimal extension from the RZM, i.e., by adding only one coupling constant to the RZM. We also examine whether this model is allowed from the viewpoint of experiments: $\mu(\tau)$ decay rate, $\mu(\tau) \rightarrow e \gamma$ [15] and neutrinoless double beta decay [39]. We briefly consider the constraints from the recent CMB observation [4, 5, 6, 7] and the possibility of the Z-burst scenario in this model [40, 41]. 
The outline of this paper is as follows: In Sec. II, we first analyze the structure of the neutrino mass matrix that is compatible with SNO/S-K and CHOOZ data. We next derive the Majorana neutrino mass matrix that is induced through the radiative correction in the GZM. The parameters of the GZM are determined by comparing the structure of the neutrino mass matrix obtained from the experiments with that obtained from the GZM. In Sec. III, we investigate the cosmological implication of the GZM. From the viewpoint of baryogenesis, there must be some combinations of the lepton numbers that avoid the washing out of the primordial baryon number. We first examine what combinations of lepton numbers are conserved in each type of GZM. We next obtain a range of parameters in which the baryon number is not washed out. We finally calculate the resulting lepton and baryon number. Section IV is devoted to a summary.

\section{THE GZM MASS MATRIX COMPATIBLE WITH THE LMA-MSW SOLU- TION}

In the present section, we review the structure of the neutrino mass matrix arising in the GZM and determine the parameters of the GZM using the neutrino oscillation data. We further examine whether the GZM is consistent with the constraints from other phenomenological experiments.

\section{A. Neutrino mass matrix that satisfies SNO/S-K and CHOOZ experimental data}

The Majorana neutrino mass term is written as

$$
\mathcal{L}_{\text {Majorana }}=\frac{1}{2} \vec{\nu}_{L}^{c} \mathcal{M}_{\nu} \vec{\nu}_{L}+\text { H.c. for } \vec{\nu}_{L} \equiv\left(\begin{array}{c}
\nu_{e} \\
\nu_{\mu} \\
\nu_{\tau}
\end{array}\right)_{L}
$$

where $\mathcal{M}_{\nu}$ is a complex valued symmetric matrix. Here, the weak eigenstate $\vec{\nu}_{L}$ is taken to diagonalize the mass matrix of the charged leptons. Using the Maki-Nakagawa-Sakata (MNS) matrix $\mathrm{U}$, we can diagonalize $\mathcal{M}_{\nu}$ to $\hat{\mathcal{M}}_{\nu}$,

$$
\hat{\mathcal{M}}_{\nu}=U^{T} \mathcal{M}_{\nu} U
$$




$$
\vec{\nu}_{L}^{m}=U^{\dagger} \vec{\nu}_{L} \equiv\left(\begin{array}{c}
\nu_{1} \\
\nu_{2} \\
\nu_{3}
\end{array}\right)_{L}
$$

where $\vec{\nu}_{L}^{m}$ is in the neutrino mass eigenstate. We write a diagonal matrix with a caret hereafter. The Lagrangian density is written in this base,

$$
\mathcal{L}_{\text {Majorana }}=\frac{1}{2}\left(\overrightarrow{\bar{\nu}}_{L}^{m}\right)^{c} \hat{\mathcal{M}}_{\nu} \vec{\nu}_{L}^{m}+\text { H.c. }
$$

We assume that $\mathcal{M}_{\nu}$ is real and the MNS matrix is orthogonal for simplicity. Under these assumptions, we can parametrize the orthogonal matrix $\mathrm{U}$ as

$$
\begin{aligned}
U & \equiv\left(\begin{array}{ccc}
1 & 0 & 0 \\
0 & c_{23} & s_{23} \\
0 & -s_{23} & c_{23}
\end{array}\right)\left(\begin{array}{ccc}
c_{13} & 0 & s_{13} \\
0 & 1 & 0 \\
-s_{13} & 0 & c_{13}
\end{array}\right)\left(\begin{array}{ccc}
c_{12} & s_{12} & 0 \\
-s_{12} & c_{12} & 0 \\
0 & 0 & 1
\end{array}\right) \\
& =R_{23}\left(-\theta_{23}\right) R_{13}\left(\theta_{13}\right) R_{12}\left(-\theta_{12}\right)
\end{aligned}
$$

where $c_{i j} \equiv \cos \theta_{i j}$ and $s_{i j} \equiv \sin \theta_{i j}$.

We summarize the current results of neutrino experiments to make clear what type of neutrino mass matrix is allowed. The $\mathrm{S}-\mathrm{K}$ experiment shows that there exist a mass squared difference $\Delta_{a}$ and a mixing angle $\theta_{a t m}$ in order to explain the atmospheric neutrino oscillation [1, 42, 43] of

$$
1.6 \times 10^{-3} \mathrm{eV}^{2}<\Delta_{a}<4.0 \times 10^{-3} \mathrm{eV}^{2}, \quad 0.88<\sin ^{2} 2 \theta_{a t m} \leq 1.0 \text { (90\% C.L.) },
$$

with the best fit values $\Delta_{a}=2.5 \times 10^{-3} \mathrm{eV}^{2}$ and $\sin ^{2} 2 \theta_{a t m}=1.00$. The global analysis of the first results of KamLAND combined with existing data from solar neutrino experiments shows that there exist a mass squared difference $\Delta_{s}$ and a mixing angle $\theta_{\odot}$ for explaining the solar neutrino oscillation [2, 3, 44, 45, 46] of

$$
\begin{gathered}
5.1 \times 10^{-5} \mathrm{eV}^{2}<\Delta_{s}<9.7 \times 10^{-5} \mathrm{eV}^{2}, \quad 1.2 \times 10^{-4} \mathrm{eV}^{2}<\Delta_{s}<1.9 \times 10^{-4} \mathrm{eV}^{2}, \\
0.29<\tan ^{2} \theta_{\odot}<0.86 \quad(3 \sigma \text { level }),
\end{gathered}
$$

with the best fit values, $\Delta_{s}=6.9 \times 10^{-5} \mathrm{eV}^{2}$ and $\tan ^{2} \theta_{\odot}=0.46$ [46]. This analysis concludes with three remarks: first, the original LMA region is narrowed and is separated into two islands (8); second, the LMA is the unique solution to $4 \sigma$ level; thirdly, the maximal solar 
neutrino mixing angle is excluded at the $3 \sigma$ level (9). These data are rewritten using the mass squared difference ratio $\mathrm{R}$ and the deviation of the solar neutrino angle from $\pi / 4$, $\theta_{\odot} \equiv \pi / 4-\epsilon_{3}$, as

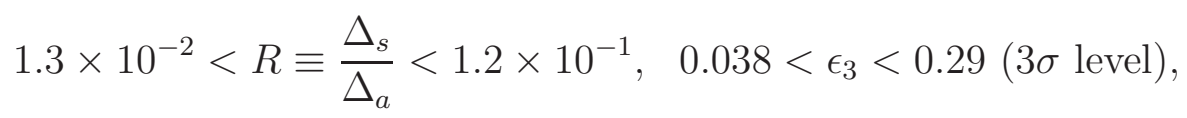

with the best fit values $R=2.8 \times 10^{-2}$ and $\epsilon_{3}=0.19$. The CHOOZ experiment shows the upper bounds for the mixing angle $\theta_{13}$ [47] to be

$$
\sin ^{2} 2 \theta_{13} \leq 0.1
$$

Since we assume that the mass matrix $\mathcal{M}_{\nu}$ is real, we can generally parametrize it as

$$
\begin{aligned}
\mathcal{M}_{\nu} & =\left(\begin{array}{rrr}
a & +b & -b \\
+b & c & d \\
-b & d & c
\end{array}\right)+\eta\left(\begin{array}{lll}
0 & 1 & 1 \\
1 & 0 & 0 \\
1 & 0 & 0
\end{array}\right)+\xi\left(\begin{array}{ccc}
0 & 0 & 0 \\
0 & 1 & 0 \\
0 & 0 & -1
\end{array}\right) \\
& \equiv \mathcal{M}_{\nu}^{0}+\mathcal{M}_{\nu}^{\eta}+\mathcal{M}_{\nu}^{\xi} .
\end{aligned}
$$

Using the orthogonal matrix

$$
U_{0} \equiv R_{23}\left(-\frac{\pi}{4}\right) R_{13}(0) R_{12}\left(-\frac{\pi}{4}+\epsilon_{3}\right) \text { with } \tan 2 \epsilon_{3}=\frac{c-a-d}{2 \sqrt{2} b},
$$

the first matrix $\mathcal{M}_{\nu}^{0}$ in Eq. (13) is diagonalized as follows [15]:

$$
\begin{aligned}
U_{0}^{T} \mathcal{M}_{\nu}^{0} U_{0} & =R_{12}\left(-\epsilon_{3}\right)\left(\begin{array}{crc}
-\sqrt{2} b+\frac{1}{2}(a+c-d) & \frac{1}{2}(a-c+d) & 0 \\
\frac{1}{2}(a-c+d) & \sqrt{2} b+\frac{1}{2}(a+c-d) & 0 \\
0 & 0 & c+d
\end{array}\right) R_{12}\left(\epsilon_{3}\right) \\
& =\left(\begin{array}{ccc}
\frac{a+c-d+\sqrt{(a-c+d)^{2}+8 b^{2}}}{2} & 0 & 0 \\
0 & \frac{a+c-d-\sqrt{(a-c+d)^{2}+8 b^{2}}}{2} & 0 \\
0 & c+d
\end{array}\right)
\end{aligned}
$$

According to the data (17) and (11), the deviations of the angles $\theta_{23}$ and $\theta_{13}$ from the values $\theta_{23}=\pi / 4$ and $\theta_{13}=0$ are small, and the influence of $\eta$ and $\xi$ is expected to be treated as a small perturbation. Defining these deviations as $\epsilon_{1} \equiv \pi / 4-\theta_{23}$ and $\epsilon_{2} \equiv \theta_{13}$, we find that they should lie in the range $0<\epsilon_{1}<0.18$ and $0<\epsilon_{2}<0.16$. We obtain the following relations within the first order approximation:

$$
\begin{aligned}
& \eta=b \epsilon_{1}-\frac{a-(d+c)}{\sqrt{2}} \epsilon_{2}, \\
& \xi=-2 d \epsilon_{1}-\sqrt{2} b \epsilon_{2} .
\end{aligned}
$$


Judging from the range of $\epsilon_{1}$ and $\epsilon_{2}$, we find that $\eta$ and $\xi$ are much smaller than the elements of $\mathcal{M}_{\nu}^{0}$. We therefore assume that $\eta$ and $\xi$ are zero in the present paper. We further assume $c=0$ for simplicity. In this case, the structure of the mass matrix belongs to the type- $\mathrm{C}$ texture with two independent zeros, defined in Ref. [31], and the phenomenology is discussed in Refs. [35, 36, 37]. We obtain the three mass eigenvalues and $\epsilon_{3}$ as follows:

$$
\begin{aligned}
\hat{M}_{\nu} \simeq U_{0}^{T} \mathcal{M}_{\nu}^{0} U_{0} & =\left(\begin{array}{ccc}
\frac{a-d+\sqrt{(a+d)^{2}+8 b^{2}}}{2} & 0 & 0 \\
0 & \frac{a-d-\sqrt{(a+d)^{2}+8 b^{2}}}{2} & 0 \\
0 & 0 & d
\end{array}\right) \equiv\left(\begin{array}{ccc}
m_{1} & 0 & 0 \\
0 & m_{2} & 0 \\
0 & 0 & m_{3}
\end{array}\right), \\
\tan 2 \epsilon_{3} & =-\frac{a+d}{2 \sqrt{2} b} .
\end{aligned}
$$

Since we identify $\Delta_{a}$ and $\Delta_{s}$ as $\Delta_{a} \equiv\left|m_{1}^{2}-m_{3}^{2}\right| \simeq\left|m_{2}^{2}-m_{3}^{2}\right|$ and $\Delta_{s} \equiv\left|m_{1}^{2}-m_{2}^{2}\right|$, we obtain the ratio $R$,

$$
R=\frac{\Delta_{s}}{\Delta_{a}}=\frac{4\left|(a-d) \sqrt{(a+d)^{2}+8 b^{2}}\right|}{\left|\left[a-d+\sqrt{(a+d)^{2}+8 b^{2}}\right]^{2}-4 d^{2}\right|} .
$$

It is shown in Ref. 21] that a must have an appropriate magnitude obtained from the experimental data mentioned above. This can be understood as follows. If we set $a=0$, we obtain a simple relation between $\tan 2 \epsilon_{3}$ and $\mathrm{R}$,

$$
\tan 2 \epsilon_{3}=\frac{1}{2 \sqrt{2}}\left|\frac{d}{b}\right| \simeq \frac{1}{4} R
$$

where $b \simeq-\sqrt{\Delta_{a} / 2}$ and $d \simeq \frac{1}{2} \sqrt{\Delta_{a}} R$. On the other hand, $\mathrm{R}$ and $\tan 2 \epsilon_{3}$ must satisfy

$$
3.2 \times 10^{-3}<\frac{1}{4} R<3.0 \times 10^{-2}, \quad 7.7 \times 10^{-2}<\tan 2 \epsilon_{3}<0.66
$$

from the data (10). We therefore find that $a$ must have an appropriate magnitude because the conditions (21) and (22) are incompatible. Similarly, we can show that $d$ must also have an appropriate magnitude. From Eqs. (19), (20), and (10), we find that mild fine-tuning $|a+d| \gg|a-d|$ is needed to fit the model with the LMA MSW solution.

Because the difference $a-d(\equiv \epsilon)$ is small in comparison with $b, a, d$, it can be treated as a small perturbation. We obtain the following values for $b, a, d$, and $\epsilon$ :

$$
\begin{aligned}
& b \simeq-\sqrt{\frac{\Delta_{a}}{2}} \simeq-3.5 \times 10^{-2} \mathrm{eV}, \\
& a \simeq\left(\tan 2 \epsilon_{3}\right) \sqrt{\Delta_{a}} \simeq 2.0 \times 10^{-2} \mathrm{eV},
\end{aligned}
$$




$$
\begin{aligned}
& d \simeq\left(\tan 2 \epsilon_{3}\right) \sqrt{\Delta_{a}}-\epsilon \simeq 1.9 \times 10^{-2} \mathrm{eV} \\
& \epsilon \simeq \frac{1}{2} \sqrt{\Delta_{a}} R \frac{1}{\sqrt{1+\left(\tan 2 \epsilon_{3}\right)^{2}}} \simeq 6.5 \times 10^{-4} \mathrm{eV}
\end{aligned}
$$

for the best fit values $\Delta_{a}=2.5 \times 10^{-3} \mathrm{eV}^{2}, \epsilon_{3}=0.19$, and $R=2.8 \times 10^{-2}$.

\section{B. GZM neutrino mass matrix}

In the present section, we review the GZM [13, 15]. The interactions of the GZM are the following:

$$
\begin{aligned}
\mathcal{L}_{\text {SM }}^{\text {Yukawa }} & =\sum_{i=1,2} \overrightarrow{\vec{l}}_{L, a} \Gamma_{i} \Phi_{i, a} \vec{e}_{R}+\text { H.c., } \\
\mathcal{L}_{\text {Zee }}^{\text {Yukawa }} & =\overrightarrow{\vec{l}}_{L, a} i\left(\sigma_{2}\right)_{a b} f \vec{l}_{L, b}^{c} h^{-}+\text {H.c. } \\
\mathcal{L}_{\text {Higic }}^{\text {cubic }} & =\lambda \Phi_{1, a}^{T} i\left(\sigma_{2}\right)_{a b} \Phi_{2, b} h^{-}+\text {H.c. }
\end{aligned}
$$

Here, $h^{-}$is the Zee singlet, $\vec{l}_{L}$ is the lepton doublet in a weak eigenstate, $\Phi_{1}$ and $\Phi_{2}$ are the two Higgs doublets, $\Gamma_{1}$ and $\Gamma_{2}$ are two Yukawa coupling matrices, $f$ is an antisymmetric coupling matrix, $\lambda$ is a cubic coupling constant, and $a, b$ are indices of the $S U(2)_{L}$ doublets.

We write the vacuum expectation value (VEV) of each Higgs doublet as $\left\langle\phi_{i}^{0}\right\rangle=v_{i} / \sqrt{2}(i$ $=1,2)$. Rotating $\Phi_{1}$ and $\Phi_{2}$ as follows:

$$
\left(\begin{array}{c}
\Phi_{1}^{\prime} \\
\Phi_{2}^{\prime}
\end{array}\right)=\left(\begin{array}{cc}
\cos \beta & -\sin \beta \\
\sin \beta & \cos \beta
\end{array}\right)\left(\begin{array}{c}
\Phi_{1} \\
\Phi_{2}
\end{array}\right)
$$

where $\tan \beta=v_{1} / v_{2}$, we make only one of the two Higgs doublets have the VEV, i.e.,

$\left\langle\phi_{1}^{\prime 0}\right\rangle=0$ and $\left\langle\phi_{2}^{\prime 0}\right\rangle=v / \sqrt{2}=\sqrt{\left(v_{1}^{2}+v_{2}^{2}\right) / 2}$. While $\phi_{2}^{\prime \pm}$ is the would-be Nambu-Goldstone boson, the diagonalization of the mass matrix for the remaining physical charged Higgs fields $h^{+}$and $\phi_{1}^{\prime}$ goes as

$$
\mathcal{L}_{\text {Higgs }} \supset\left(h^{+}, \phi_{1}^{\prime+}\right)\left(\begin{array}{rr}
M_{h}^{2} & -\frac{\lambda v}{\sqrt{2}} \\
-\frac{\lambda v}{\sqrt{2}} & \left(M_{1}^{\prime+}\right)^{2}
\end{array}\right)\left(\begin{array}{c}
h^{-} \\
\phi_{1}^{\prime-}
\end{array}\right)=\left(S_{1}^{+}, S_{2}^{+}\right)\left(\begin{array}{rr}
M_{1}^{2} & 0 \\
0 & M_{2}^{2}
\end{array}\right)\left(\begin{array}{c}
S_{1}^{-} \\
S_{2}^{-}
\end{array}\right),
$$

using the orthogonal transformation

$$
\left(\begin{array}{c}
S_{1}^{-} \\
S_{2}^{-}
\end{array}\right)=\left(\begin{array}{cc}
\cos \alpha & -\sin \alpha \\
\sin \alpha & \cos \alpha
\end{array}\right)\left(\begin{array}{c}
h^{-} \\
\phi_{1}^{\prime-}
\end{array}\right)
$$


where the rotational angle $\alpha$ and the two mass eigenvalues $M_{1}, M_{2}$ are determined as

$$
\begin{aligned}
\tan 2 \alpha & =\frac{-\sqrt{2} \lambda v}{\left(M_{1}^{\prime+}\right)^{2}-M_{h}^{2}}, \\
\left(M_{1,2}\right)^{2} & =\frac{M_{h}^{2}+\left(M_{1}^{\prime+}\right)^{2} \pm \sqrt{\left[M_{h}^{2}-\left(M_{1}^{\prime+}\right)^{2}\right]^{2}+2 \lambda^{2} v^{2}}}{2} .
\end{aligned}
$$

Using $\Phi_{1}^{\prime}$ and $\Phi_{2}^{\prime}$ in Eq. (30), the Lagrangian density (27) is written as

$$
\mathcal{L}_{S M}^{Y \text { Yukawa }}=\vec{l}_{L, a}\left[\left(\cos \beta \Gamma_{1}-\sin \beta \Gamma_{2}\right) \Phi_{1, a}^{\prime}+\left(\sin \beta \Gamma_{1}+\cos \beta \Gamma_{2}\right) \Phi_{2, a}^{\prime}\right] \vec{e}_{R}+\text { H.c. }
$$

Since only $\phi_{2}^{\prime 0}$ has the VEV, using the biunitary transformation

$$
\begin{aligned}
\vec{l}_{L, a}^{m} & \equiv V_{L} \vec{l}_{L, a}, \vec{l}_{L, 1}^{m} \equiv \vec{\nu}_{L}=\left(\begin{array}{c}
\nu_{e} \\
\nu_{\mu} \\
\nu_{\tau}
\end{array}\right)_{L}, \vec{l}_{L, 2}^{m} \equiv \vec{e}_{L}^{m}=\left(\begin{array}{c}
e \\
\mu \\
\tau
\end{array}\right)_{L}, \\
\vec{e}_{R}^{m} & \equiv V_{R} \vec{e}_{R}=\left(\begin{array}{c}
e \\
\mu \\
\tau
\end{array}\right)_{R} .
\end{aligned}
$$

The matrix $\left(\sin \beta \Gamma_{1}+\cos \beta \Gamma_{2}\right)$ is diagonalized as

$$
\hat{\Gamma}^{m} \equiv V_{L}\left(\sin \beta \Gamma_{1}+\cos \beta \Gamma_{2}\right) V_{R}^{\dagger} \equiv \frac{\sqrt{2}}{v} \hat{M}_{l-m}=\frac{\sqrt{2}}{v}\left(\begin{array}{ccc}
m_{e} & 0 & 0 \\
0 & m_{\mu} & 0 \\
0 & 0 & m_{\tau}
\end{array}\right),
$$

where $\hat{M}_{l-m}$ is the mass matrix of the charged leptons. The Lagrangian density (27) is written as

$$
\mathcal{L}_{S M}^{\text {Yukawa }}=\overrightarrow{\vec{l}}_{L, a}^{m}\left[\left(\cos \beta \Gamma_{1}^{m}-\sin \beta \Gamma_{2}^{m}\right) \Phi_{1, a}^{\prime}+\hat{\Gamma}^{m} \Phi_{2, a}^{\prime}\right] \vec{e}_{R}^{m}+\text { H.c. },
$$

where $\Gamma_{i}^{m} \equiv V_{L} \Gamma_{i} V_{R}^{\dagger}(i=1,2)$.

Since we estimate the interaction rates in the early Universe in Sec. III we write down the Lagrangian density in the symmetric phase $(v=0)$ in addition to that in the symmetry broken phase $(v=246 \mathrm{GeV})$ where the neutrinos have the Majorana masses.

1. Symmetric phase $(v=0)$

The interaction Lagrangian density is as follows:

$$
\begin{aligned}
\mathcal{L}_{S M}^{Y \text { ukawa }} & =\overrightarrow{\bar{l}}_{L, a}^{m}\left[\left(\frac{1}{\tan \beta} \hat{\Gamma}^{m}-\frac{1}{\sin \beta} \Gamma_{2}^{m}\right) \Phi_{1, a}^{\prime}+\hat{\Gamma}^{m} \Phi_{2, a}^{\prime}\right] \vec{e}_{R}^{m}+\text { H.c. } \\
\mathcal{L}_{\text {Zee }}^{Y \text { ukawa }} & =2 \overrightarrow{\bar{\nu}}_{L}^{m} f^{m}\left(\vec{e}_{L}^{m}\right)^{c} h^{-}+\text {H.c. } \\
\mathcal{L}_{\text {Higis }}^{\text {cubic }} & =\lambda \Phi_{1, a}^{\prime T} i\left(\sigma_{2}\right)_{a b} \Phi_{2, b}^{\prime} h^{-}+\text {H.c. }
\end{aligned}
$$


Here, all leptons are massless and $f^{m}\left(\equiv V_{L} f V_{L}^{T}\right)$ is antisymmetric.

2. Symmetry broken phase $(v=246 \mathrm{GeV})$

The interaction Lagrangian density is as follows:

$$
\begin{aligned}
\mathcal{L}_{S M}^{Y \text { ukawa }}= & \overrightarrow{\bar{\nu}}_{L}^{e}\left[\left(\frac{1}{\tan \beta} \hat{\Gamma}^{m}-\frac{1}{\sin \beta} \Gamma_{2}^{m}\right)\left(-\sin \alpha S_{1}^{+}+\cos \alpha S_{2}^{+}\right)+\hat{\Gamma}^{m} \phi_{2}^{\prime+}\right] \vec{e}_{R}^{m} \\
& +\overrightarrow{\bar{e}}_{L}^{m}\left[\left(\frac{1}{\tan \beta} \hat{\Gamma}^{m}-\frac{1}{\sin \beta} \Gamma_{2}^{m}\right) \phi_{1}^{\prime 0}+\hat{\Gamma}^{m}\left(\frac{v+\sigma+i N_{2}}{\sqrt{2}}\right)\right] \vec{e}_{R}^{m}+\text { H.c. } \\
\mathcal{L}_{\text {Zee }}^{\text {unawa }}= & 2 \overrightarrow{\bar{\nu}}_{L}^{m} f^{m}\left(\vec{e}_{L}^{m}\right)^{c}\left(\cos \alpha S_{1}^{-}+\sin \alpha S_{2}^{-}\right)+\text {H.c. }, \\
\mathcal{L}_{\text {Higgs }}^{\text {cubic }}= & \lambda\left(\cos \alpha S_{1}^{-}+\sin \alpha S_{2}^{-}\right)\left[\frac{1}{\sqrt{2}}\left(\sigma+i N_{2}\right)\left(-\sin \alpha S_{1}^{+}+\cos \alpha S_{2}^{+}\right)\right. \\
& \left.-\phi_{1}^{\prime 0} \phi_{2}^{\prime+}\right]+ \text { H.c. }
\end{aligned}
$$

Here, the Higgs fields $S_{1}^{-}, S_{2}^{-}$have masses $M_{1}, M_{2}$, respectively. The fields $\phi_{2}^{\prime}$ and $N_{2}$ are the would-be Nambu-Goldstone bosons.

In this phase, the Majorana neutrino mass is calculated from the two one-loop diagrams in Fig. 11 and the transposed diagrams of them, for $m_{e}, m_{\mu}, m_{\tau} \ll M_{1}, M_{2}$,

$$
\begin{aligned}
i \mathcal{M}^{\alpha \beta} & =A\left[\left(f^{*} \hat{M}_{l}^{2}+\hat{M}_{l}^{2} f^{\dagger}\right)-\frac{v}{\sqrt{2} \cos \beta}\left(f^{*} \hat{M}_{l} \Gamma_{2}^{m \dagger}+\Gamma_{2}^{m *} \hat{M}_{l} f^{\dagger}\right)\right]^{\alpha \beta} \\
( & \left.=i \mathcal{M}^{T^{\alpha \beta}}\right),
\end{aligned}
$$

where $A \equiv\left(1 / 8 \sqrt{2} \pi^{2} v \tan \beta\right) \sin 2 \alpha \log \left(M_{2}^{2} / M_{1}^{2}\right)$ and $\gamma_{2}^{\alpha \beta}, \hat{\gamma}^{\alpha \beta}$ are elements of the matrices $\Gamma_{2}^{m}, \hat{\Gamma}^{m}$, respectively.

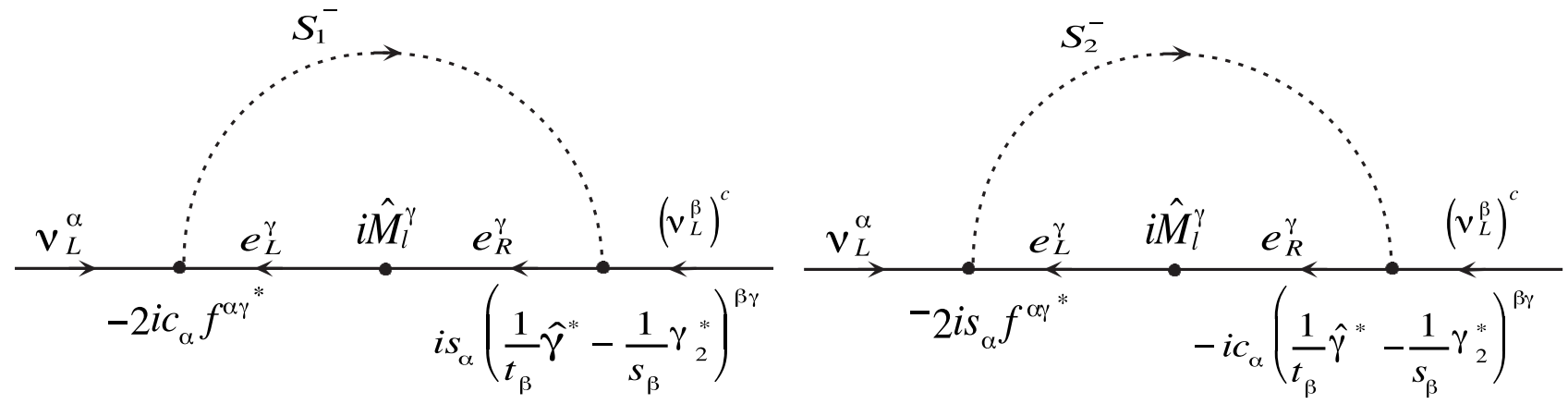

FIG. 1: The diagrams that are used to calculate the radiatively induced neutrino mass matrix. We use $s_{\alpha} \equiv \sin \alpha, c_{\alpha} \equiv \cos \alpha$, and $t_{\alpha} \equiv \tan \alpha$ and so on in this figure. 


\section{Matching the GZM with the experiments}

We obtained the relation between the value of $a$ and the experimental data in Sec. IA.

$$
a=\tan 2 \epsilon_{3} \sqrt{\Delta_{a}}
$$

On the other hand, we calculated the radiatively induced $a$ in the GZM in Sec. IIB,

$$
a=-2 A B\left[m_{\mu} f^{* e \mu} \gamma_{2}^{* e \mu}+m_{\tau} f_{2}^{* e \tau} \gamma_{2}^{* e \tau}\right]
$$

where $B=v / \sqrt{2} \cos \beta$. From Eq. (47) and Eq. (48), we find that one of $\gamma_{2}^{e \tau}$ and $\gamma_{2}^{e \mu}$ must be nonzero at least. Because we prefer a minimal extension here, we assume that the other elements of $\Gamma_{2}^{m}$ are zero. From now on, we further assume that $f$ and $\Gamma_{2}^{m}$ are real. Here, we match the neutrino mass matrix in the GZM (46) with that determined from the experimental data (23) $-(25)$ as follows

$$
\begin{aligned}
& b=-\sqrt{\frac{\Delta_{a}}{2}}=A\left[f^{e \mu} m_{\mu}^{2}-B m_{\tau} f^{\mu \tau} \gamma_{2}^{e \tau}\right]=-A\left[f^{e \tau} m_{\tau}^{2}+B m_{\mu} f^{\mu \tau} \gamma_{2}^{e \mu}\right] \\
& a=\tan 2 \epsilon_{3} \sqrt{\Delta_{a}}=-2 A B\left[m_{\mu} f^{e \mu} \gamma_{2}^{e \mu}+m_{\tau} f^{e \tau} \gamma_{2}^{e \tau}\right] \\
& d=\tan 2 \epsilon_{3} \sqrt{\Delta_{a}}-\epsilon=A f^{\mu \tau} m_{\tau}^{2}
\end{aligned}
$$

In this model, there are nine parameters $\left(M_{1}, M_{2}, \alpha, \beta, f^{e \mu}, f^{e \tau}, f^{\mu \tau}, \gamma_{2}^{e \tau}, \gamma_{2}^{e \mu}\right)$. From Eqs. (49) - (151), we parametrize four parameters $\left(f^{e \mu}, f^{e \tau}, f^{\mu \tau}, \gamma_{2}^{e \tau}\right)$ using the other five parameters $\left(M_{1}, M_{2}, \alpha, \beta, \gamma_{2}^{e \mu}\right)$ as follows

$$
\begin{aligned}
f^{e \mu} & =\frac{1}{A} \frac{b\left[b+B d\left(m_{\mu} / m_{\tau}^{2}\right) \gamma_{2}^{e \mu}\right]+\frac{1}{2} d a}{m_{\mu}^{2}\left[b+B d\left(m_{\mu} / m_{\tau}^{2}\right) \gamma_{2}^{e \mu}\right]-B d m_{\mu} \gamma_{2}^{e \mu}} \\
f^{e \tau} & =-\frac{b}{A m_{\tau}^{2}}-\frac{B}{A} d \frac{m_{\mu}}{m_{\tau}^{4}} \gamma_{2}^{e \mu}, \\
f^{\mu \tau} & =\frac{d}{A m_{\tau}^{2}}, \\
\gamma_{2}^{e \tau} & =\frac{m_{\tau}}{m_{\mu}} \frac{b \gamma_{2}^{e \mu}+(a / 2 B) m_{\mu}}{b+B d \gamma_{2}^{e \mu}\left(1 / m_{\tau}^{2}-1 / m_{\mu}^{2}\right)} .
\end{aligned}
$$

We consider the simplest two cases, $(1) \gamma_{2}^{e \tau} \neq 0, \gamma_{2}^{e \mu}=0$ and $(2) \gamma_{2}^{e \tau}=0, \gamma_{2}^{e \mu} \neq 0$. In these cases, we have the following relations.

(1) $\gamma_{2}^{e \tau} \neq 0, \gamma_{2}^{e \mu}=0$. Equations (52) $-($ (55) are written as

$$
f^{e \mu}=\frac{b}{A m_{\mu}^{2}}+\frac{d a}{2 A b m_{\mu}^{2}} \simeq-3.7 \times 10^{-5}\left(\frac{10^{-4} \mathrm{GeV}^{-1}}{A}\right),
$$




$$
\begin{aligned}
& f^{e \tau}=-\frac{b}{A m_{\tau}^{2}} \simeq 1.1 \times 10^{-7}\left(\frac{10^{-4} \mathrm{GeV}^{-1}}{A}\right) \\
& f^{\mu \tau}=\frac{d}{A m_{\tau}^{2}} \simeq 6.1 \times 10^{-8}\left(\frac{10^{-4} \mathrm{GeV}^{-1}}{A}\right), \gamma_{2}^{e \tau}=\frac{a m_{\tau}}{2 B b} \simeq-2.1 \times 10^{-3}\left(\frac{\cos \beta}{1 / \sqrt{2}}\right) .
\end{aligned}
$$

(2) $\gamma_{2}^{e \tau}=0, \gamma_{2}^{e \mu} \neq 0$. Equations (52)-(5.5) are written as

$$
\begin{aligned}
f^{e \mu} & =\frac{b}{A m_{\mu}^{2}} \simeq-3.2 \times 10^{-5}\left(\frac{10^{-4} \mathrm{GeV}^{-1}}{A}\right) \\
f^{e \tau} & =-\frac{b}{A m_{\tau}^{2}}+\frac{m_{\mu}^{2}}{m_{\tau}^{4}} \frac{d a}{2 A b} \simeq-\frac{b}{A m_{\tau}^{2}} \simeq 1.1 \times 10^{-7}\left(\frac{10^{-4} \mathrm{GeV}^{-1}}{A}\right), \\
f^{\mu \tau} & =\frac{d}{A m_{\tau}^{2}} \simeq 6.1 \times 10^{-8}\left(\frac{10^{-4} \mathrm{GeV}^{-1}}{A}\right), \gamma_{2}^{e \mu}=-\frac{a m_{\mu}}{2 B b} \simeq 1.2 \times 10^{-4}\left(\frac{\cos \beta}{1 / \sqrt{2}}\right) .
\end{aligned}
$$

\section{Experimental constraints on $\tau(\mu)$ decay, $\tau(\mu) \rightarrow \mathbf{e} \gamma$ and $0 \nu \beta \beta$ amplitude}

In the present section, we examine whether the parameters obtained in the previous section [Eqs. (56), (57)] are consistent with the constraints from the processes $\tau(\mu)$ decay, $\tau(\mu) \rightarrow \mathrm{e} \gamma$, and $0 \nu \beta \beta$ decay [15].

1. $\tau(\mu)$ decay

Since the leptonic flavor changing neutral current does not exist in the standard model at all, stringent constraints are imposed on their decay amplitudes [48]. There is, however, $\tau(\mu)$ decay through the FCNCs at the tree level in the GZM. These constraints are satisfied for the parameter set (56) and (57) as follows for $\beta \simeq \pi / 4$ :

$$
\begin{aligned}
\operatorname{Br}\left(\tau^{-} \rightarrow e^{-} \mu^{-} \mu^{+}\right) \simeq \frac{\left|\gamma_{2}^{e \tau}\left(\sqrt{2} m_{\mu} / v\right)\right|^{2}}{G_{F}^{2}\left(M_{1}^{\prime 0}\right)^{4}} & \lesssim 1.1 \times 10^{-10} \\
& \ll 1.8 \times 10^{-6} \text { (experiment [48]), } \\
\operatorname{Br}\left(\mu^{-} \rightarrow e^{-} e^{-} \mu^{+}\right) \simeq \frac{\left|\gamma_{2}^{e \mu}\left(\sqrt{2} m_{e} / v\right)\right|^{2}}{G_{F}^{2}\left(M_{1}^{\prime 0}\right)^{4}} & \lesssim 8.7 \times 10^{-18} \\
& \ll 1.0 \times 10^{-12} \text { (experiment [48]), }
\end{aligned}
$$

where $G_{F}$ is the Fermi coupling constant and $M_{1}^{\prime 0} \gtrsim 100 \mathrm{GeV}$. Since the coupling constants $f^{e \mu}, f^{e \tau}$, and $f^{\mu \tau}$ are much smaller than $\gamma_{2}^{e \tau}$ and $\gamma_{2}^{e \mu}$, the contributions to the branching ratios purely from $f$ are negligible [49, 50, 51].

2. $\tau(\mu) \rightarrow \mathrm{e} \gamma$ 
Since these processes violate the lepton flavor, they do not exist in the standard model at all. On the other hand, these processes become possible in the GZM through the exchange of the charged and neutral Higgs bosons. Since they are rare events, stringent constraints are imposed on the amplitudes 48]. These constraints are safely satisfied for the parameter set (56) and (57) as follows for $\beta \simeq \pi / 4$ :

$$
\begin{aligned}
& \operatorname{Br}\left(\tau^{-} \rightarrow e^{-} \gamma\right) \simeq \alpha \frac{\left|\gamma_{2}^{e \tau}\left(\sqrt{2} m_{\tau} / v\right)\right|^{2}}{G_{F}^{2}\left(M^{\prime}{ }_{1}\right)^{4}} \lesssim 2.3 \times 10^{-10} \ll 2.7 \times 10^{-6} \text { (experiment [48]), } \\
& \operatorname{Br}\left(\mu^{-} \rightarrow e^{-} \gamma\right) \simeq \alpha \frac{\left|\gamma_{2}^{e \mu}\left(\sqrt{2} m_{\mu} / v\right)\right|^{2}}{G_{F}^{2}\left(M_{1}^{\prime 0}\right)^{4}} \lesssim 2.8 \times 10^{-15} \ll 1.2 \times 10^{-11} \text { (experiment [48]). }
\end{aligned}
$$

Again, since the coupling constants $f^{e \mu}, f^{e \tau}$, and $f^{\mu \tau}$ are much smaller than $\gamma_{2}^{e \tau}$ and $\gamma_{2}^{e \mu}$, the contributions to the branching ratios purely from $f$ are negligible [49, 50, 51].

\section{3. $0 \nu \beta \beta$}

The observation of the neutrinoless double beta decay $(0 \nu \beta \beta)$ gives evidence that the neutrinos have Majorana masses. The study of this process is a crucial test of the validity of our model, since neutrinos have Majorana masses, the interaction of the Zee singlet $h^{-}$violates lepton number, and the Yukawa coupling $\Gamma_{2}^{m}$ is flavor changing. As was discussed by Schechter and Valle [52] in the theory with an exotic doubly charged scalar, the extension of the Higgs sector possibly provides a new source of the decay. We can, in fact, think of a process with the exchange of our exotic scalar, i.e., $h^{-}$[see Fig. 2(a)]. However, we readily know that this diagram does not exist in our model. This is because the Yukawa coupling of $h^{-}$is flavor off-diagonal and the intermediate neutrinos are either $\nu_{\mu}$ or $\nu_{\tau}$. This, in turn, means that the Yukawa coupling of $\phi_{1}^{\prime-}$ at another vertex should be flavor changing, i.e., $\gamma_{2}^{\mu e}$ or $\gamma_{2}^{\tau e}$, which vanishes in our model. Another source of the double beta decay comes from the exchange of Majorana neutrinos with Majorana mass insertion due to $W^{ \pm}$exchange. As was first pointed out by Wolfenstein [53], the amplitude is proportional to $m_{e e}$. The absence of any report of this process so far gives the upper bounds to the element $m_{e e}$ in the neutrino mass matrix [48] [60],

$$
\left|m_{e e}\right|<0.1 \mathrm{eV}(90 \% \text { C.L. }) .
$$


The value obtained in Sec. $\llbracket \mathrm{A}$ is consistent with this upper bound as follows:

$$
m_{e e}=a \simeq 2.0 \times 10^{-2} \mathrm{eV}
$$

Since the Yukawa coupling $\Gamma_{2}^{m}$ is flavor changing, another source of decay exists as shown in Fig. 2(b), where $m_{\mu \mu}$ or $m_{\tau \tau}$ contributes, but not $m_{e e}$. This diagram, however, does not exist from reasoning similar to the above; namely, the amplitude of the diagram is also proportional to $\gamma_{2}^{\mu e}$ or $\gamma_{2}^{\tau e}$, which does not exist. Thus the ordinary process due to $m_{e e}$ discussed above is the unique source of double beta decay. If the sensitivity of the experiment is improved by a factor of 5 or so, the decay should be seen if our model is valid.

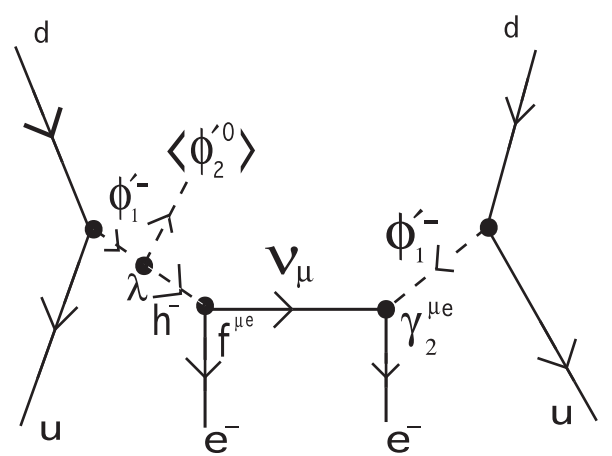

(a)

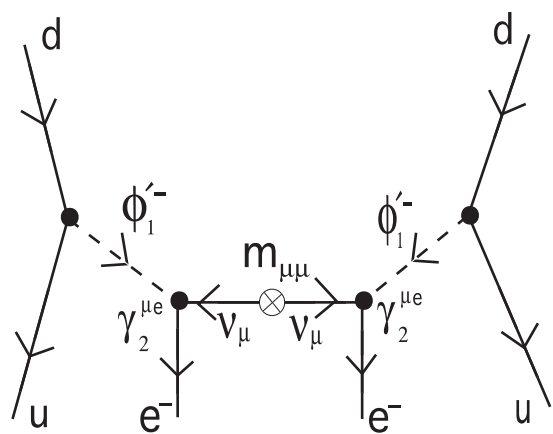

(b)

FIG. 2: The diagrams contributing to neutrinoless double beta decay. In (a), the $h^{-}$interaction violates the lepton number, while in (b) the lepton number is violated by the insertion of $m_{\mu \mu}$. Both processes fail to exist because of the vanishing Yukawa coupling $\gamma_{2}^{\mu e}=0$. In both diagrams $\nu_{\mu}$ may be replaced by $\nu_{\tau}$.

\section{COSMOLOGICAL IMPLICATIONS OF THE GZM}

In Sec. III, we obtained the values for the coupling constants $\left(f^{e \mu}, f^{e \tau}, f^{\mu \tau}, \gamma_{2}^{e \tau}, \gamma_{2}^{e \mu}\right)$ suggested by the neutrino oscillation data. In the present section, we investigate whether the primordial baryon number is washed out or not for these values. If there are some leptonic conserved numbers, the primordial baryon number remains finite even though the sphaleron 
process partially washes out the baryon number. We therefore consider what leptonic numbers are effectively conserved at a high temperature. We then calculate what amount of the primordial baryon number remains in the case that there exist some conserved numbers. We also briefly consider the constraints from the recent CMB observation [4, 5, 6, 7] and the possibility of the Z-burst scenario in this model [40, 41].

\section{A. Conserved number for each coupling}

It is convenient to define the following global $U(1)$ charges as

$$
L_{e \mu} \equiv L_{\tau}-L_{e}-L_{\mu}, \quad L_{e \tau} \equiv L_{\mu}-L_{e}-L_{\tau}, \quad L_{\mu \tau} \equiv L_{e}-L_{\mu}-L_{\tau}
$$

for an investigation into the washing out of the baryon number. These quantities are conserved numbers in the standard model. We, however, have coupling constants which violate these conserved numbers in our model. Each coupling conserves two of the three independent numbers which are shown in the Table \using a linear combination of $L_{e \mu}, L_{e \tau}$, and $L_{\mu \tau}$.

\begin{tabular}{|c|c|c|c|c|c|}
\hline Coupling constant & $f^{e \mu}$ & $f^{e \tau}$ & $f^{\mu \tau}$ & $\gamma_{2}^{e \tau}$ & $\gamma_{2}^{e \mu}$ \\
\hline Conserved number & $L_{e \tau}, L_{\mu \tau}$ & $L_{e \mu}, L_{\mu \tau}$ & $L_{e \mu}, L_{e \tau}$ & $L_{e \tau}, L_{e \mu}+L_{\mu \tau}$ & $L_{e \mu}, L_{e \tau}+L_{\mu \tau}$ \\
\hline
\end{tabular}

TABLE I: Conserved number for each coupling constant.

\section{B. Interaction rates induced by each coupling}

We here estimate the interaction rates induced by the coupling constants $f$ and $\gamma_{2}$.

1. $L_{\mu \tau}$ violating process induced by the coupling constant $f^{\mu \tau}$

We consider the $h^{-}$decay process and the inverse decay process induced by the coupling constant $f^{\mu \tau}$. The interaction rates for these processes are estimated at a temperature $\mathrm{T}$ $\gtrsim M_{h}[27,56]$,

$$
\Gamma\left(h^{-} \leftrightarrow \mu^{-} \nu_{\tau}\right)=\frac{1}{2 \pi}\left|f^{\mu \tau}\right|^{2} \frac{M_{h}^{2}}{E} \simeq \frac{1}{5.4 \pi}\left|f^{\mu \tau}\right|^{2} \frac{M_{h}^{2}}{T},
$$

where $M_{h}$ is the mass of the Zee singlet and $\langle E\rangle \simeq 2.7 \mathrm{~T}$ (boson) is the averaged energy. Similar results are obtained for the processes induced by the coupling constants $f^{e \mu}$ and $f^{e \tau}$. 


\section{Processes induced by the coupling constant $\gamma_{2}^{e \tau}$}

We next estimate the interaction rates for the processes induced by the coupling constant $\gamma_{2}^{e \tau}$. We estimate the interaction rates of $\phi_{1}^{\prime-}$ decay and the inverse decay. The interaction rate for the process $\phi_{1}^{\prime-} \leftrightarrow\left(\nu_{L}^{e}\right)^{c} \tau_{R}^{-}$is estimated for $\mathrm{T} \gtrsim M_{1}^{\prime+}$,

$$
\Gamma\left(\phi_{1}^{\prime-} \leftrightarrow\left(\nu_{L}^{e}\right)^{c} \tau_{R}^{-}\right) \simeq \frac{1}{22 \pi}\left|\frac{\gamma_{2}^{e \tau}}{\sin \beta}\right|^{2} \frac{\left(M_{1}^{\prime+}\right)^{2}}{T}
$$

where $M_{1}^{\prime+}$ is the mass of the field $\phi_{1}^{\prime-}$. Similar results are obtained for the processes induced by the coupling constant $\gamma_{2}^{e \mu}$.

\section{Out-of-equilibrium condition to avoid the baryon number washing out}

In the present section, we consider which processes discussed in the previous section were out of equilibrium in the early Universe [61]. We will consider what numbers are conserved in the case where these processes are out of equilibrium in the next section. The condition that the processes are out of equilibrium is given by

$$
\Gamma<H
$$

where $\mathrm{H}$ is the Hubble parameter, $H=1.66 \sqrt{g_{*}} T^{2} / M_{P l} \simeq 1.47 \times 10^{-18}\left(T^{2} / \mathrm{GeV}\right)$ with $M_{P l}$ being the Planck mass. Here, $g_{*}$ is the total degrees of freedom of effectively massless particles. In the GZM, we adopt the value $g_{*}=g_{*}^{S M}+g_{*}^{h^{-}}+g_{*}^{\Phi_{2}} \simeq 112.75$.

1. Coupling constants $f^{e \mu}, f^{e \tau}$, and $f^{\mu \tau}$ are out of equilibrium

$f^{\mu \tau}$ process. We examine the condition that the process $h^{-} \leftrightarrow \mu^{-} \nu_{\tau}$ is out of equilibrium at $T=M_{h}$. We apply the condition (65) to the rate (63),

$$
\begin{aligned}
& \Gamma\left(h^{-} \leftrightarrow \mu^{-} \nu_{\tau}\right)<H \\
& \Longleftrightarrow \frac{1}{5.4 \pi}\left|f^{\mu \tau}\right|^{2} \frac{M_{h}^{2}}{T}<1.5 \times 10^{-18}\left(\frac{T^{2}}{\mathrm{GeV}}\right) .
\end{aligned}
$$

We obtain the condition for $T=M_{h}$,

$$
2.9 \times 10^{6} \frac{\tan ^{4} \beta}{\sin ^{4} 2 \alpha} \mathrm{GeV}^{2}<\left|\log \frac{M_{1}^{2}}{M_{2}^{2}}\right|^{4}\left[M_{1}^{2}+M_{2}^{2}+\left(M_{1}^{2}-M_{2}^{2}\right) \cos 2 \alpha\right],
$$


where we use the following relations:

$$
\begin{aligned}
\left|f^{\mu \tau}\right| & =\left|\frac{d}{A m_{\tau}^{2}}\right|, A=\frac{1}{8 \sqrt{2} \pi^{2} v \tan \beta} \sin 2 \alpha\left|\log \frac{M_{1}^{2}}{M_{2}^{2}}\right|, \\
M_{h}^{2} & =\frac{M_{1}^{2}+M_{2}^{2}+\left(M_{1}^{2}-M_{2}^{2}\right) \cos 2 \alpha}{2} .
\end{aligned}
$$

At the higher temperatures, $T>M_{h}$, the out-of-equilibrium condition Eq. (67) is more easily satisfied. At lower temperatures, $T<M_{h}$, the number density of the Zee singlet $h^{-}$ in the thermal bath is so small that the $L_{\mu \tau}$-violating processes are negligible. It is therefore sufficient to consider the out-of-equilibrium condition at $\mathrm{T} \simeq M_{h}$. We set $\beta=\pi / 4$ for simplicity hereafter. The inequality (68) is written for this value of $\beta$,

$$
\frac{2.9 \times 10^{6}}{\sin ^{4} 2 \alpha} \mathrm{GeV}^{2}<\left(\log \frac{M_{1}^{2}}{M_{2}^{2}}\right)^{4}\left[M_{1}^{2}+M_{2}^{2}+\left(M_{1}^{2}-M_{2}^{2}\right) \cos 2 \alpha\right] .
$$

From Eq. (71), we find that this condition is easily satisfied for the angle $\sin 2 \alpha \sim 1$. We then fix the value of $\alpha$ to $\pi / 4$. In this case, the inequality (71) is simplified as

$$
18<\left(\log \frac{y}{x}\right)^{4}\left[x^{2}+y^{2}\right]
$$

where we parametrize as $x \equiv M_{1} / 100 \mathrm{GeV}, y \equiv M_{2} / 100 \mathrm{GeV}(x, y \geq 1, x \geq y)$. From this, we find that this process is out of equilibrium for $M_{1} \geq 310 \mathrm{GeV}$, when we fix the value of $M_{2}$ to $100 \mathrm{GeV}$ [62].

$f^{e \tau}$ process. The out-of-equilibrium condition for the $f^{e \tau}$ process is obtained just as the condition for the $f^{\mu \tau}$ process. We examine the condition that the process $h^{-} \leftrightarrow e^{-} \nu_{\tau}$ is out of equilibrium at $T=M_{h}$. We apply the condition (65) to the rate (63),

$$
\begin{aligned}
& \frac{1}{5.4 \pi}\left|f^{e \tau}\right|^{2} \frac{M_{h}^{2}}{T}<1.47 \times 10^{-18}\left(\frac{T^{2}}{\mathrm{GeV}}\right) \\
& \Longleftrightarrow \frac{2.9 \times 10^{7}}{\sin ^{4} 2 \alpha} \mathrm{GeV}^{2}<\left(\log \frac{M_{1}^{2}}{M_{2}^{2}}\right)^{4}\left[M_{1}^{2}+M_{2}^{2}+\left(M_{1}^{2}-M_{2}^{2}\right) \cos 2 \alpha\right] .
\end{aligned}
$$

In the case $\alpha=\pi / 4$, the inequality (174) is simplified as

$$
1.8 \times 10^{2}<\left(\log \frac{y}{x}\right)^{4}\left[x^{2}+y^{2}\right]
$$

From this, we find that this process is out of equilibrium for $M_{1} \geq 500 \mathrm{GeV}$, when we fix the value of $M_{2}$ to $100 \mathrm{GeV}$. 
$f^{e \mu}$ process. The out-of-equilibrium condition for the $f^{e \mu}$ process is obtained in a similar manner to that in the $f^{\mu \tau}$ process. The condition $\Gamma\left(h^{-} \leftrightarrow e^{-} \nu_{\mu}\right)<H$ is written as

$$
\frac{2.0 \times 10^{17}}{\sin ^{4} 2 \alpha} \mathrm{GeV}^{2}<\left(\log \frac{M_{1}^{2}}{M_{2}^{2}}\right)^{4}\left[M_{1}^{2}+M_{2}^{2}+\left(M_{1}^{2}-M_{2}^{2}\right) \cos 2 \alpha\right] \text {. }
$$

From this, we find that this process is out of equilibrium for $M_{1} \geq 1.2 \times 10^{6} \mathrm{GeV}$, when we fix the value of $\alpha$ to $\pi / 4$ and the value of $M_{2}$ to $100 \mathrm{GeV}$. Since we recognize that this mass range for $M_{1}$ is quite large and somewhat unnatural, we consider that this process is in equilibrium at $T=M_{h}$.

\section{Coupling constants $\gamma_{2}^{e \tau}$ and $\gamma_{2}^{e \mu}$ are out of equilibrium}

We consider which processes induced from the coupling constants $\gamma_{2}^{e \tau}$ and $\gamma_{2}^{e \mu}$ are out of equilibrium for each case considered in Sec. 【IC $\gamma_{2}^{e \tau} \neq 0, \gamma_{2}^{e \mu}=0$ and $\gamma_{2}^{e \tau}=0, \gamma_{2}^{e \mu} \neq 0$.

$\gamma_{2}^{e \tau} \neq 0$ and $\gamma_{2}^{e \mu}=0$. We examine the condition that the process $\phi_{1}^{\prime-} \leftrightarrow\left(\nu_{L}^{e}\right)^{c} \tau_{R}^{-}$is out of equilibrium at $T=M_{h}$,

$$
\begin{aligned}
& \Gamma\left(\phi_{1}^{\prime-} \leftrightarrow\left(\nu_{L}^{e}\right)^{c} \tau_{R}^{-}\right)<H, \\
& \Rightarrow 8.4 \times 10^{10} \mathrm{GeV}<\sqrt{\frac{M_{1}^{2}+M_{2}^{2}}{2}},
\end{aligned}
$$

where we fix the value of $\alpha$ to $\pi / 4$. From this, we find that this process is out of equilibrium for $M_{1} \geq 1.2 \times 10^{11} \mathrm{GeV}$, when we fix the value of $M_{2}$ to $100 \mathrm{GeV}$. We consider that this process is in equilibrium at $T=M_{h}$ for the same reason mentioned above.

$\gamma_{2}^{e \tau}=0$ and $\gamma_{2}^{e \mu} \neq 0$. We examine the condition that the process $\phi_{1}^{\prime-} \leftrightarrow\left(\nu_{L}^{e}\right)^{c} \mu_{R}^{-}$is out of equilibrium as follows:

$$
\begin{aligned}
& \Gamma\left(\phi_{1}^{\prime-} \leftrightarrow\left(\nu_{L}^{e}\right)^{c} \mu_{R}^{-}\right)<H, \\
& \Rightarrow 2.8 \times 10^{8} \mathrm{GeV}<\sqrt{\frac{M_{1}^{2}+M_{2}^{2}}{2}},
\end{aligned}
$$

where we fix the value of $\alpha$ to $\pi / 4$. From this, we find that this process is out of equilibrium for $M_{1} \geq 4.0 \times 10^{8} \mathrm{GeV}$, when we fix the value of $M_{2}$ to $100 \mathrm{GeV}$. We consider that this process is also in equilibrium at $T=M_{h}$.

\section{Final lepton number and baryon number}

In the present section, we consider the final lepton and baryon number for the two cases $\gamma_{2}^{e \tau} \neq 0, \gamma_{2}^{e \mu}=0$ and $\gamma_{2}^{e \tau}=0, \gamma_{2}^{e \mu} \neq 0$ 


$$
\text { 1. } \gamma_{2}^{e \tau} \neq 0, \gamma_{2}^{e \mu}=0
$$

Since the processes induced by $\gamma_{2}^{e \tau}$ and $f^{e \mu}$ are in equilibrium from the previous section, the only possible conserved quantity is

$$
P \equiv L_{e \tau}
$$

This quantity is conserved if the process induced by $f^{e \tau}$ is out of equilibrium [Eq. (174)]. The region in which this quantity is conserved is shown in Fig. 3. This conserved number is converted to the baryon number through the sphaleron process and the final baryon $\left(B_{f}^{e q}\right)$ and lepton number $\left(L_{f}^{e q}\right)$ are calculated as

$$
\begin{aligned}
B_{f}^{e q} & =\frac{60}{563}\left(\frac{B}{3}+L_{e \tau}\right)_{i}, \\
L_{f}^{e q} & =-\frac{39}{20} B_{f}^{e q}
\end{aligned}
$$

where $\left(B / 3+L_{e \tau}\right)_{i}$ is the primordial value generated at the temperature much higher than $T=M_{h}$. The region shown in Fig. 3 is therefore the allowed region for avoiding the washing out of the primordial baryon number in this case.

$$
\text { 2. } \gamma_{2}^{e \tau}=0, \gamma_{2}^{e \mu} \neq 0
$$

Since the processes induced by $\gamma_{2}^{e \mu}$ and $f^{e \mu}$ are in equilibrium, the only possible conserved quantity in this case is

$$
P \equiv L_{e \tau}+L_{\mu \tau}\left(=-2 L_{\tau}\right)
$$

This quantity is conserved if the process induced from $f^{e \tau}$ and $f^{\mu \tau}$ is out of equilibrium [Eq. (74) and Eq. (711)]. The region in which this quantity is conserved is shown in Fig. 3. This conserved number is converted to the baryon number through the sphaleron process and the final baryon $\left(B_{f}^{e q}\right)$ and lepton number $\left(L_{f}^{e q}\right)$ are calculated as

$$
\begin{aligned}
B_{f}^{e q} & =\frac{60}{185}\left(\frac{B}{3}-L_{\tau}\right)_{i}, \\
L_{f}^{e q} & =-\frac{39}{20} B_{f}^{e q},
\end{aligned}
$$

where $\left(B / 3-L_{\tau}\right)_{i}$ is a primordial value generated at the temperature much higher than $T=M_{h}$. The region shown in FIG. 3 is therefore the allowed region for avoiding the washing out of the primordial baryon number in this case, also. 


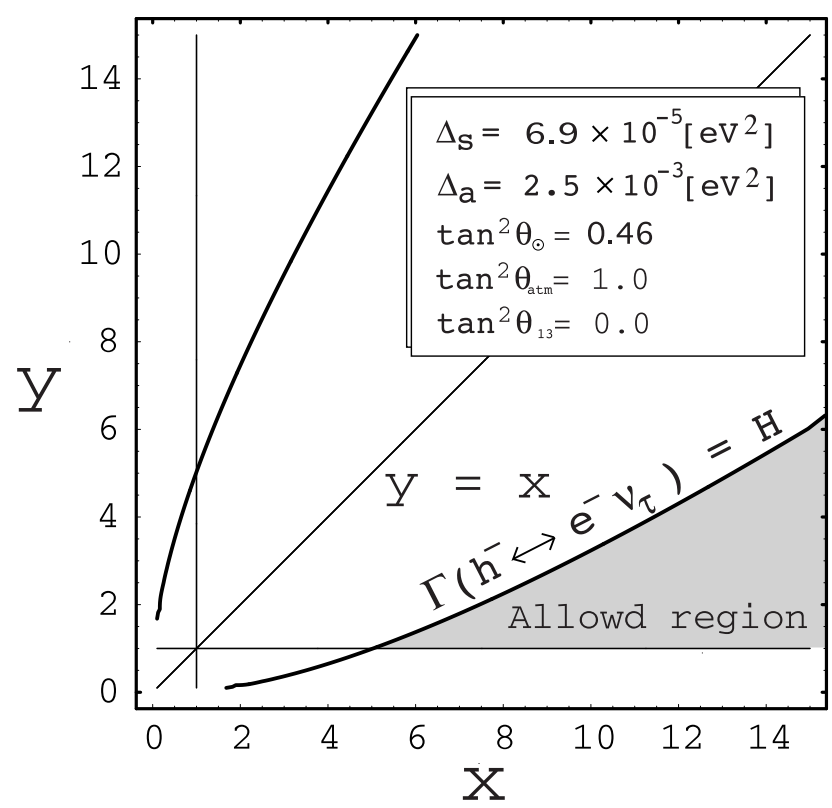

FIG. 3: The primordial baryon number remains finite in the allowed region. The out-of-equilibrium condition $1.8 \times 10^{2}<[\log (y / x)]^{4}\left[x^{2}+y^{2}\right][$ Eq. (75)] is used in this figure with the variables $x \equiv M_{1} / 100 \mathrm{GeV}, y \equiv M_{2} / 100 \mathrm{GeV}$. Only the region $x \geq 1, y \geq 1, x \geq y$ is considered.

\section{E. WMAP and Z burst}

The investigation into the cosmic microwave background by the Wilkinson Microwave Anisotropy Probe (WMAP) recently gave the following upper bound to the sum of the neutrino masses [4, 5, 6 , 7]:

$$
\sum_{i}\left|m_{i}\right|<0.71 \mathrm{eV}(95 \% \text { C.L. }) .
$$

This value is consistent with that obtained in Sec. 【A.

$$
\sum_{i}\left|m_{i}\right|=\sqrt{(2 a-\epsilon)^{2}+8 b^{2}}+d \simeq 0.13 \mathrm{eV} .
$$

If the ultrahigh energy cosmic rays with energy beyond the Greisen-Zatsepin-Kuzmin limit are explained in the Z-burst scenario, the heaviest neutrino mass should lie in the range [40, 41, 57]

$$
0.08 \mathrm{eV}<\mid m_{\nu} \text { (heaviest) } \mid<1.3 \mathrm{eV} \text { (68\% C.L.) }
$$

The value obtained in Sec. ஹA.

$$
\left|m_{1}\right|=\frac{\epsilon+\sqrt{(2 a-\epsilon)^{2}+8 b^{2}}}{2} \simeq 5.3 \times 10^{-2} \mathrm{eV},
$$


is near this range, and the ultrahigh energy cosmic rays might be explained in the Z-burst scenario in our model.

\section{SUMMARY}

We propose a simple GZM which is compatible with all the neutrino oscillation data for the LMA MSW solution of the solar neutrino problem, and is free from the baryon number washing out. Our extension from the RZM is minimal, i.e., we add only one coupling constant $\gamma_{2}^{e \tau}$ or $\gamma_{2}^{e \mu}$ in addition to those in the RZM. To avoid the baryon number washing out, the masses $M_{1}$ and $M_{2}$ must be in the region shown in Fig. 3, The final baryon and lepton numbers are calculated for the two cases $(1) \gamma_{2}^{e \tau} \neq 0, \gamma_{2}^{e \mu}=0$ and $(2) \gamma_{2}^{e \tau}=0, \gamma_{2}^{e \mu} \neq 0$ :

$$
\begin{aligned}
& \text { (1) } \gamma_{2}^{e \tau} \neq 0, \gamma_{2}^{e \mu}=0: B_{f}^{e q}=\frac{60}{563}\left(\frac{B}{3}+L_{e \tau}\right)_{i}, L_{f}^{e q}=-\frac{39}{20} B_{f}^{e q}, \\
& \text { (2) } \gamma_{2}^{e \tau}=0, \gamma_{2}^{e \mu} \neq 0: B_{f}^{e q}=\frac{60}{185}\left(\frac{B}{3}-L_{\tau}\right)_{i}, L_{f}^{e q}=-\frac{39}{20} B_{f}^{e q} .
\end{aligned}
$$

In order to explain today's baryon number, it is therefore necessary that the quantity $(B / 3+$ $\left.L_{e \tau}\right)$ or $\left(B / 3-L_{\tau}\right)$ is generated in the early Universe or the baryon number should be produced at lower temperatures $T \ll M_{h}$. We check that the predicted lepton flavor violating processes are not in conflict with the phenomenological constraints for $\tau(\mu)$ decay, $\tau(\mu) \rightarrow$ $e \gamma$, and $0 \nu \beta \beta$ decay.

\section{Acknowledgments}

We thank N. Haba for fruitful discussions and for providing us with the detailed calculation of Ref. [27]. One of the author (K.O.) thanks the Japan society for the promotion of science for financial support (No.4834). The work of C.S.L. was supported in part by

a Grant-in-Aid for Scientific Research of the Ministry of Education, Science, and Culture, Grant No.80201870.

[1] Super-Kamiokande Collaboration, Y. Fukuda et al., Phys. Rev. Lett. 81, 1562 (1998); 82, 2644 (1999); S. Fukuda et al., ibid. 85, 3999 (2000).

[2] SNO Collaboration, Q.R. Ahmad et al., Phys. Rev. Lett. 87, 071301 (2001). 
[3] KamLAnD Collaboration, K. Eguchi et al., Phys. Rev. Lett. 90, 021802 (2003).

[4] C.L. Bennett et al., astro-ph/0302207,

[5] D.N. Spergel et al., astro-ph/0302209.

[6] A. Pierce and H. Murayama, hep-ph/0302131.

[7] G. Bhattacharyya, H. Pas, L. Song, and T.J. Weiler, Phys. Lett. 564, 175 (2003).

[8] S. Hannestad, astro-ph/0303076

[9] Ø. Elgarøy and O. Lahav, J. Cosmos. Astropart. Phys. 04, 004 (2003)

[10] T. Yanagida, in Proceedings of the Workshop on the Unified Theory and Baryon Number in the Universe, Tsukuba, Japan, edited by O. Sawada and A. Sugamoto, KEK report No. 7918,1979, p. 95 .

[11] M. Gell-Mann, P. Rammond and R. Slansky, in Supergravity, edited by P. van Niewenhuizen and D. Z. Freedman (North Holland, Amsterdam, 1979), p.315.

[12] R. N. Mohapatra and G. Senjanovic, Phys. Rev. Lett. 44, 912 (1980).

[13] A. Zee, Phys. Lett. 93B, 389 (1980).

[14] L. Wolfenstein, Nucl.Phys. B175, 93 (1980).

[15] K.R.S. Balaji, W. Grimus, and T. Schwetz, Phys. Lett. B 508, 301 (2001).

[16] C. Jarlskog, M. Matsuda, S. Skadhauge, and M. Tanimoto, Phys. Lett. B 449, 240 (1999).

[17] P.H. Frampton and S.L. Glashow, Phys. Lett. B 461, 95 (1999).

[18] Y. Koide, Phys. Rev. D 64, 077301 (2001).

[19] J.T. Peltoniemi and J.W.F. Valle, Nucl. Phys. B406, 409 (1993).

[20] P. Roy and S.K. Vempati, Phys. Rev. D 65, 073011 (2002).

[21] P.H. Frampton, M.C. Oh, and T. Yoshikawa, Phys. Rev. D 65, 073014 (2001).

[22] J. Schechter and J.W.F. Valle, Phys. Rev. D 22, 2227 (1980).

[23] T. Kitabayashi and M. Yasue, Phys. Lett. B 524, 308 (2002), Int. J. Mod. Phys. A 17, 2519 (2002).

[24] P.H. Frampton, M.C. Oh, and T. Yoshikawa, Phys. Rev. D 66, 033007 (2002).

[25] E. Ma, M. Raidal, and U. Sarkar, Phys. Lett. B 460, 359 (1999).

[26] U. Sarkar, Pramana 54, 101 (2000).

[27] N. Haba, K. Hamaguchi, and T. Suzuki, Phys. Lett. B 519, 243 (2001).

[28] V.A. Kuzmin, V.A. Rubakov, and M.E. Shaposhnikov, Phys. Lett. B 155, 36 (1985).

[29] A.G. Cohen, D.B. Kaplan, and A.E. Nelson, Annu. Rev. Nucl. Part. Sci.43, 27, (1993). 
[30] J.T. Peltoniemi and J.W.F. Valle, Phys. Lett. B 304, 147 (1993).

[31] P.H. Frampton, S.L. Glashow, and D. Marfatia, Phys. Lett. B 536, 79 (2002).

[32] G.C. Branco, R.G. Felipe, E.R. Joaquim, and T. Yanagida, Phys. Lett. B 562, 265 (2003).

[33] A. Zee, Phys. Lett. 161B, 141 (1985); Nucl.Phys. B264, 99 (1986).

[34] K. S. Babu, Phys. Lett. B 203, 132 (1988).

[35] Z.Z. Xing, Phys. Lett. B 530, 159 (2002).

[36] Z.Z. Xing, Phys. Lett. B 539, 85 (2002).

[37] B.R. Desai, D.P. Roy, and A.R. Vaucher, Mod. Phys. Lett. A 18, 1335 (2003).

[38] T. Hambye, Nucl.Phys. B 633, 171 (2002).

[39] M.Y. Cheng and K. Cheung, hep-ph/0203051.

[40] T.J. Weiler, Astropart. Phys. 11, 303 (1999).

[41] D. Fargion et al., Astrophys. J. 517, 725 (1999).

[42] N. Fornengo, M.C. Gonzalez-Garcia, and J.W.F. Valle, Nucl. Phys. B580, 58 (2000); T. Toshito, hep-ex/0105023

[43] M.C. Gonzalez-Garcia, M. Maltoni, C. Pena-Garay, and J.W.F. Valle, Phys. Rev. D 63, 033005 (2001).

[44] J.N. Bahcall, M.C. Gonzalez-Garcia, and C. Pene-Garay, J High Energy Phys. 08, 014 (2001);

V. Barger, D. Marfatia, K. Whisnant, and B.P. Wood, Phys. Lett. B 537, 179 (2002).

[45] J.N. Bahcall, M.C. Gonzalez-Garcia, and C. Pene-Garay, J High Energy Phys. 02, 009 (2003)

[46] M. Maltoni, T. Schwetz, and J.W.F. Valle, Phys. Rev. D 67, 093003 (2003).

[47] CHOOZ Collaboration, M. Apollonio et al., Phys. Lett. B 420, 397 (1998); B 466, 415 (1999).

[48] Particle Data Group, D.E. Groom et al., Eur. Phys. J. B 15, 1 (2000).

[49] S. T. Petcov, Phys. Lett. 115B, 401 (1982).

[50] A. Y. Smirnov and M. Tanimoto, Phys. Rev. D 55, 1665 (1997).

[51] E. Mitsuda and K. Sasaki, hep-ph/0103202.

[52] J. Schechter and J.W.F. Valle, Phys. Rev. D 25, 2951 (1982).

[53] L. Wolfenstein, Phys. Lett. 107B, 77 (1981).

[54] H.V. Klapdor-Kleingrothaus et al., Mod. Phys. Lett. A 16, 2409 (2002).

[55] C. E. Aalseth et.al., Mod. Phys. Lett. A 17, 1475 (2002).

[56] E. W. Kolb and M. S. Turner, The Early Universe (Addison-Wesley, Reading, MA, 1990).

[57] Z. Fodor, S. D. Katz, and A. Ringwald, J High Energy Phys. 06, 046 (2002); Y. Fukuda et 
al., Phys. Rev. Lett. 81, 1562 (1998).

[58] We note that the constraint from the baryon number washing out is also avoided by baryogenesis at a temperature lower than the scale of the singlet Higgs boson mass, e.g., electroweak baryogenesis [28, 29] or low temperature leptogenesis [38]. The order of the electroweak phase transition can be altered due to the additional Higgs boson scalar field [30]. On the other hand, low temperature leptogenesis is impossible in the model we propose in this paper because of the absence of $\mathrm{CP}$ violation and an adequate nonequilibrium. Some additional extensions are needed for it.

[59] We do not consider how the primordial baryon number is produced. Baryogenesis in an extended version of the Zee model is discussed in Ref. [38].

[60] It was recently reported that the first evidence for $0 \nu \beta \beta$ has been observed [54]. The value is $\left|m_{e e}\right|=0.11 \sim 0.56 \mathrm{eV}(95 \%$ C.L. $)$ with the best fit value $0.39 \mathrm{eV}$. There are also, however, arguments against this report [55].

[61] Strictly speaking, scattering processes like $\mu^{-} \nu_{\tau} \leftrightarrow \phi_{1}^{0 c} \phi_{2}^{-}$may be in equilibrium for very large $\lambda$. We do not consider such a case, however.

[62] In Ref. 27], it is difficult for the LMA MSW solution to satisfy the out-of-equilibrium condition because the value of the angle $\alpha$ is set to $0.01 \mathrm{rad}$. 